\title{
Libro: La enseñanza de la Ciencia Política en las universidades de América Latina \\ Autores Varios
}

Red Latinoamericana de Carreras de Ciencia Política (RE.LA.Cl.P), Programa de Promoción de la Universidad Argentina, Secretaría de Políticas Universitarias, Ministerio de Educación Editorial Ciudad Gótica, Rosario, 2010 (256 pp.)

\section{Gisela Signorelli}

Docente de la Facultad de Ciencia Política y Relaciones Internacionales de la Universidad Nacional de Rosario y Becaria Doctoral del Consejo Nacional de Investigaciones Científicas y Técnicas, Argentina.

E-mail: giselav_signorelli@yahoo.com.ar

En los años ' 80 se fortalecen la Ciencia Política y la Teoría Política como campos académicos y universitarios así como su relación con el mundo de la política, dado que ha emergido un gran tema dador de sentido: la democracia (Lesgart, 2007). Con el retorno a la democracia, se empieza a generar en la Argentina -según señalan Bulcourf y D'Alessandro (2003)- un proceso de institucionalización y profesionalización de la Ciencia Política.

Sin embargo, junto con este crecimiento de la disciplina -tanto en el campo científico como de inserción profesional (aunque a un ritmo más pausado que en el primero' ${ }^{1}$ - la reflexión sobre su desarrollo en las últimas tres décadas y sobre sus potencialidades en el siglo XXI es generalmente escasa o muy restringida. Asimismo, no son usuales los debates sobre el estado del arte en la disciplina (Lesgart, 2007: 122) ni sobre uno de los principales desafíos que la misma enfrenta: la innovación en la enseñanza a futuros profesionales de los saberes centrales de la politología.

Es evidente que la enseñanza de la Ciencia Política y las Relaciones Internacionales requiere de un replanteo sustantivo y de un compromiso serio tanto por parte de las instituciones como de los propios docentes (Bulcourf, 2008: 241).

En ese camino se inscribe la compilación de artículos presentes en el libro La enseñanza de la Ciencia Política en las universidades de América Latina, de la Red Latinoamericana de Carreras de Ciencia Política (RE.LA.CI.P). La Red aborda las problemáticas propias de la Ciencia Política a partir de la distinción de tres áreas temáticas específicas: Teoría Política, Política Comparada y Análisis Político.

Dicha Red -dirigida desde la Universidad Nacional de Entre Ríos-, cuenta con la participación de la Universidad Nacional de Rosario, sede de la Unidad de Gestión del proyecto, y suma a nivel latinoamericano a la Universidad de Río Grande Do Sul (Brasil) y a la Universidad de la República (Uruguay). ${ }^{2}$

Como fruto de un valioso intercambio entre docentes de dichas Casas de Estudio, en este libro se reúnen aportes en las tres áreas temáticas descriptas con el 
objeto de sistematizar las experiencias de docentes con trayectoria en la enseñanza de la Ciencia Política. Aunque con una orientación a un público lector que se esté iniciando en dicha disciplina, el recorrido realizado por los autores contribuye también a la discusión respecto del recorte y recorrido de la misma tanto como de las dificultades que deben ser sorteadas en las próximas décadas respecto al lugar que se desea que la Ciencia Política ocupe entre las Ciencias Sociales, comenzando por innovar en el espacio áulico y de formación de nuevos profesionales.

Con la mirada puesta en ello, en la Primera Parte, dedicada al área de la Teoría Política, se abordan dos tipos de problemas: la dificultad de definir la teoría política por un lado, y la discusión respecto de "lo político" y "la política", por el otro. Aparece también, en los artículos de la Prof. Billoni, de la Lic. Branda, del Dr. Giavedoni, del Lic. Moreira, el Mg. Mutti y la Mg. Porcel (todos docentes de Teoría Política) la controversia entre las conceptualizaciones de la teoría y la filosofía política junto a la noción de ciencia -o el problema de la cientificidad de la Ciencia Política - así como las disputas de sentido entre la Teoría Política y la Historia. Es allí donde se manifiesta un issue importante en la definición de la disciplina y de su objeto, a partir de la construcción conceptual y el recorrido por una tradición de discurso propia de la Ciencia Política (sobre todo a partir de la lectura de los clásicos).

Por otra parte, en la misma sección, se puede encontrar el aporte del Prof. Gantus, respecto de la centralidad que recobraron en las últimas décadas las instituciones de la mano del neoinstitucionalismo, cuya máxima es tomar en serio a las instituciones formales como reglas del juego social.

La Segunda Parte del libro, está dedicada a la Política Comparada. Con aportes del equipo de dicha cátedra de la Universidad Nacional de Rosario, de Daniel Chasquetti -de la Universidad de La República de Uruguay- y de André Marenco por parte de la Universidade Federal do Rio Grande do Sul (Brasil). En los tres casos se realiza una descripción del recorrido de la Política Comparada en los países de donde son oriundos y más precisamente de su inclusión en los programas de enseñanza de las carreras de Ciencia Política respectivas, ya sea a nivel de grado o de posgrado. Se coincide en el estado incipiente de investigaciones comparativas aunque también en el progresivo avance del área en el interior de la disciplina, predominando como subcampo autónomo de conocimiento.

Por último, la Tercera Parte incluye artículos relacionados con el Análisis Político. La Prof. Cristina Díaz introduce el apartado reconstruyendo la historia -a partir de su experiencia docente- de la enseñanza del área en el interior de la carrera de Ciencia Política en la Universidad Nacional de Rosario, así como sus desafíos actuales a través de las decisiones y mecanismos plasmados en estrategias pedagógicas para desentrañar y construir los sentidos de la coyuntura en el momento áulico (pp. 171). Los demás artículos que constituyen la sección realizan un análisis de la labor de algunos analistas reconocidos de los medios gráficos nacionales. Las Lic. Di Filippo y Pinto toman las editoriales de Joaquín Morales Solá y Mariano Grondona durante la reconocida "crisis del campo" de 2008 en Argentina. Por su parte, Lo Valvo y Martínez, realizan una lectura del análisis de Mario 
Wainfeld bajo la misma coyuntura política. Finalmente, la Lic. Gisela Scaglia y el Prof. Barberis problematizan el legado de la historia en las visiones del momento actual, a partir de un estudio del suplemento: "Peronismo. Filosofía política de una obstinación argentina", de José Pablo Feinmann, publicado en 2007 por el diario Página 12.

El problema de la Ciencia Política -afirma Gastón Mutti (p.81)- se reduce a su inmadurez, que la lleva a la dispersión y a la heterogeneidad. Este libro es un reflejo de ello, se trata de una ciencia amplia, que resguarda para sí estos tres campos - la Teoría Política, la Política Comparada y el Análisis Político- entre otros, pero todos y cada uno con la mirada siempre puestas en el poder, la política y el Estado.

La enseñanza de la Ciencia Política en las universidades de América Latina es, en fin, un recorrido heterogéneo, complejo pero sumamente interesante que cuestiona, analiza y se interroga sobre las prácticas del oficio de enseñar la Ciencia Política, demostrando que además de una sólida formación se requiere algo de arte (Bulcourf, 2008) para aggionarse a lo que el nuevo siglo requiere de sus profesionales.

\section{Referencias}

1. Para mayor desarrollo al respecto puede consultarse la segunda tesis trabajada por Leiras, Abal Medina y D'Alessandro (2005).

2. Información extraída de http://unr.edu.ar/noticia/3380/observatorio-de-acciones-internacionalesentrevista-a-miembros-de-la-relacip.

\section{Bibliografía}

P. BULCOURF (2003), "Desarrollos recientes en la ciencia política argentina”, en VV. AA., ponencia presentada en el VI Congreso Nacional de Ciencia Política, SAAP, Rosario.

P. BULCOURF (2008), "Algunas reflexiones sobre la enseñanza de Ciencia Política en la Argentina”, en PosData, Nro. 13.

Y. CHEVALLARD (1997), La transposición didáctica. Del saber sabio al saber enseñado, Buenos Aires, Aique.

C. LESGART (2007), "Pasado y presente de la Ciencia Política producida en Argentina. Aportes para un debate sobre su porvenir", en Temas y Debates, Rosario, Año 11, № 14.

M. LEIRAS, J. M. ABAL MEDINA Y M. D'ALESSANDRO (2005), "La ciencia política en la Argentina: el camino de la institucionalización dentro y fuera de las aulas universitarias", Revista de Ciencia Política, Santiago de Chile, Vol. 25, $\mathrm{N}^{\circ} 1$.

\section{Otras fuentes}

Observatorio de Acciones Internacionales - Entrevista a miembros de la RELACIP. Disponible en: http://unr.edu.ar/noticia/3380/observatorio-de-acciones-internacionales-entrevista-a-miembros-de-larelacip. Publicado el 4/7/2011.. Consultado el 15/2/2013.

Entrevista a Cristina Díaz en UNER Noticias, Periódico digital de la Universidad Nacional de Entre Ríos. Disponible en: http://www.noticias.uner.edu.ar/node/1697. Consultado el 15/2/2013. 